\title{
Notes préliminaires sur la distribution spatiale de Meta menardi, Triphosa dubitata, Triphosa sabaudiata, Nelima aurantiaca* et Culex pipiens au sein d'un écosystème cavernicole (Grotte de la Scierie: Hte.-Savoie)
}

by

\author{
John D. BOURNE**
}

\begin{abstract}
SUMMARY
A preliminary note on the spatial distribution of Meta menardi, Triphosa dubitata, Triphosa sabaudiata, Nelima aurantiaca and Culex pipiens within a cave ecosystem (Grotte de la Scierie: Haute-Savoie)
\end{abstract}

Following 8 months of observations in a richly populated cave, (Grotte de la Scierie, HauteSavoie, France), it has been possible to outline the movements and distribution of Meta menardi (Araneae), Nelima aurantiaca (Opiliones), Triphosa duhitata and Trinhosa sabaudiata (Lepidoptera) and Culex pipiens (Diptera) within the cave ecosystem. Although no general rule can be postulated it appears that the morphology of the cave walls and the climatic conditions regulate the distribution of these 5 species within the cave ecosystem. The interactions between the 5 species have been investigated.

\section{INTRODUCTION}

Malgré l'intérêt croissant que suscite le milieu pariétal pour les biospéologues, il n'existe à ce jour aucune étude quantitative sur les relations spatiales entre les membres de cette biocénose. Dans le cadre d'une étude à longue échéance d'écologie comparative entre deux grottes d'une même vallée, cette première note analyse la biocénose pariétale d'une des grottes. Les cinq espèces (l'araignée Meta menardi Latreille, les papillons Triphosa dubitata L. et Triphosa sabaudiata Duponchel, l'opilion Nelima aurantiaca Simon et le moustique Culex pipiens L.) sont bien représentés dans cette cavité. La

* Nom récemment changé: Amilenus aurantiacus.

** Adresse de l'auteur: route du Jura 1261 LA RIPPE (Suisse). 
Grotte de la Scierie a été choisie pour cette raison et aussi parce qu'elle possède une morphologie simple.

Certaines des espèces choisies pour cette analyse ont été les sujets d'études passées mais prises individuellement. En 1933, déjà, Roubaud démontrait que Culex pipiens accomplit une diapause hivernale dans les grottes où les conditions climatologiques jouent un rôle déterminant. La distribution spatiale au sein du biotope des araignées Meta menardi selon Tercafs (1972) se fait au hasard mais elle est contagieuse dans l'écosystème souterrain. Dans leur article très intéressant sur les papillons cavernicoles, Bouvet et Coll. (1974) donnent des informations biologiques et écologiques pour Triphosa dubitata et Triphosa sabaudiata. En effet ces papillons accomplissent une diapause hivernale dans les grottes du Jura méridional et ont tendance à former des "agrégats" dans leur biotope. Ce dernier phénomène a été investigué par Graham (1968) pour le papillon sub-troglophile des EtatsUnis, Triphosa haesitata. J'ai également noté la tendance chez T. sabaudiata à former des "agrégats" dans les biotopes mis en évidence par ce travail (voir résultats).

\section{MATÉRIELS ET MÉTHODES}

La Grotte de la Scierie (fig. 1) se trouve sur le versant nord de la montagne de Bange à 650 mètres d'altitude et l'entrée, orientée au nord, est ombragée

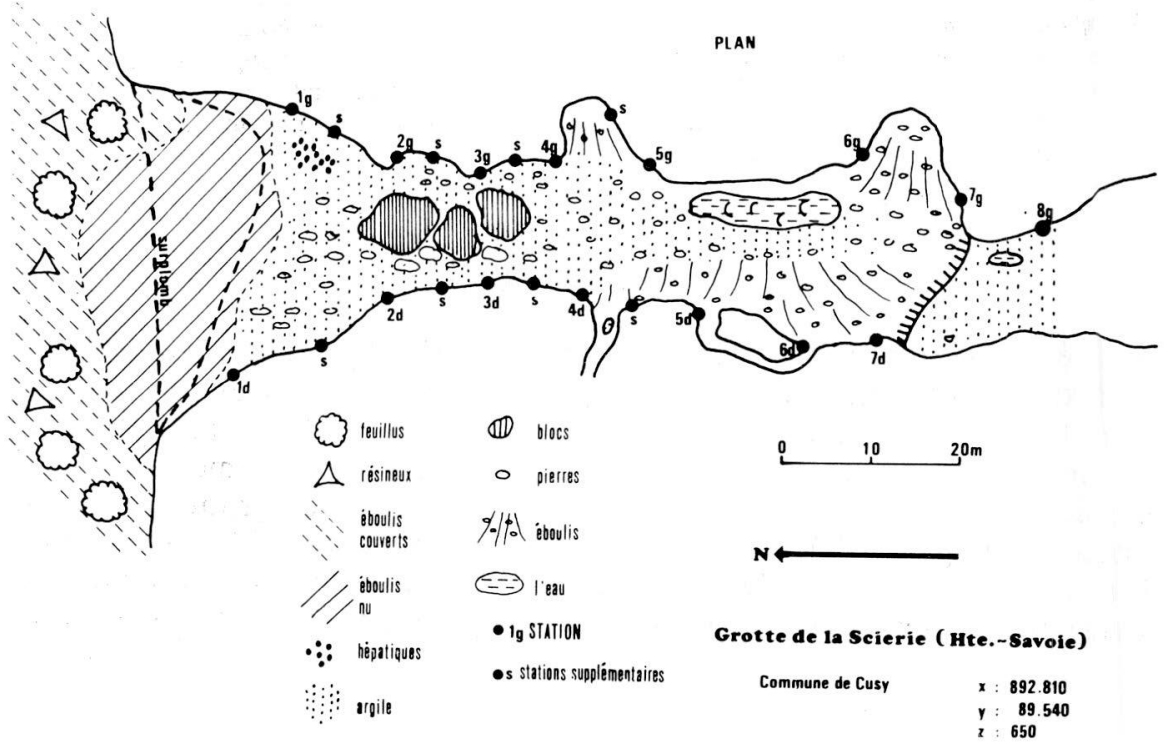

Fig. 1. Plan de la Grotte de la Scierie montrant les positions des stations. 
par un important surplomb. L'entrée ne reçoit jamais de soleil direct. Le milieu forestier extérieur est du type hygrophile avec des feuillus et résineux en nombre égale et un sous-bois composé de fougères, mousses et hépatiques. La composition climatologique, floristique, faunistique et géographique du milieu proche de l'entrée est sûrement décisive pour la qualité et la quantité de l'association pariétale.

\begin{tabular}{|c|c|c|c|c|c|c|c|}
\hline Stations & 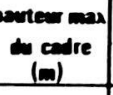 & eriemation & Inmiere & Statious & 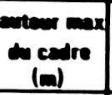 & orrientation & Imieniere \\
\hline $1 \mathrm{~g}$ & 2,5 & & DIRECTE & $1 d$ & 2,0 & & DIRECTE \\
\hline $2 g$ & 1,5 & & DIFFUSE & $2 d$ & 1,0 & & FolRECTE \\
\hline $3 g$ & 1,0 & & MUt & $3 d$ & 1,0 & & DIFFUSE \\
\hline $4 g$ & 1,0 & & MUL & $4 d$ & 1,0 & & NUL \\
\hline $5 g$ & 1,5 & & WUL & $5 d$ & 4,5 & & NUL \\
\hline $6 \mathrm{~g}$ & 2,5 & & MUL & $6 d$ & 5,0 & & NUL \\
\hline $7 g$ & 1,5 & & MUL & $7 d$ & 4,5 & & MUL \\
\hline $8 g$ & 4,5 & & WUI & & & 1 & \\
\hline
\end{tabular}

Fig. 2. Morphologie des parois et facteur lumière pour les 15 stations. 
A cause de l'importance numérique de la faune, une quinzaine de cadres d'un mètre carré furent posés selon la figure 1. Chacun des cadres était composé de quatre petits cadres de $0,25 \mathrm{~m}^{2}$. Lors de chaque visite (au moins une fois par mois), les positions des cinq espèces à l'intérieur des cadres furent notées de même que la température et l'humidité au milieu du cadre. Pour s'assurer que la représentation de la biomasse délimitée par un cadre était plus ou moins typique pour sa position au sein de l'écosystème, une estimation de la faune environnante était faite à chaque visite. Ceci s'est révélé juste dans tous les cas sauf pour la station $6 \mathrm{~g}$ (voir résultats). L'orientation des deux espèces de papillons vis-à-vis de l'entrée de la grotte fut également relevée.

La description morphologique de chaque biotope délimité par les cadres est résumée dans la figure 2 de même que l'influence de la lumière et la hauteur du côté supérieur du cadre par rapport au sol de l'entrée ( 0 mètre).

En plus des comptages des papillons dans les cadres, 98 positions hors des cadres furent notées afin d'obtenir une analyse plus juste de l'orientation de ces insectes.

Les affinités entre chaque espèce furent calculées par l'emploi de la formule simple de Sorensen:

$$
q=\frac{2 c}{a+b} \times 100
$$

où $a$ est égal au nombre de relevés renfermant l'espèce $\mathrm{A}, b$ au nombre de relevés renfermant l'espèce $B$ et $c$ au nombre de relevés qui contiennent simultanément les deux espèces.

\section{CLIMATOLOGIE}

La figure 3 résume les variations de température et d'humidité pendant les 8 mois qu'a duré l'étude (oct. 1974 à mai 1975) et n'a qu'une valeur comparative. Des données plus précises seront publiées plus tard dans le cadre d'une comparaison de cette cavité avec la Grotte de Bange. Les températures pour les stations $4 \mathrm{~g}$ et $4 \mathrm{~d}$ qui ont été prises avec un thermomètre maximum/minimum et comparées avec les relevés mensuels directs ne montraient que les différences minimales. Cependant il est à noter que les variations à l'extérieur pourraient être plus marquées. La hauteur d'une station par rapport à l'entrée (fig. 2) révèle les raisons pour lesquelles les stations $5 \mathrm{~d}, 6 \mathrm{~d}, 7 \mathrm{~d}$ et $8 \mathrm{~g}$ sont en général plus chaudes.

Les mouvements d'air dans cette cavité ne sont pas évidents mais les mouvements de convection créés par les différences de température (entre le plafond et le sol et entre le fond et l'extérieur) sont à noter. Une forte condensation est évidente entre les stations 5 et 8 à partir de 5 mètres du sol: pendant le printemps les gouttes d'eau tombent du plafond. 

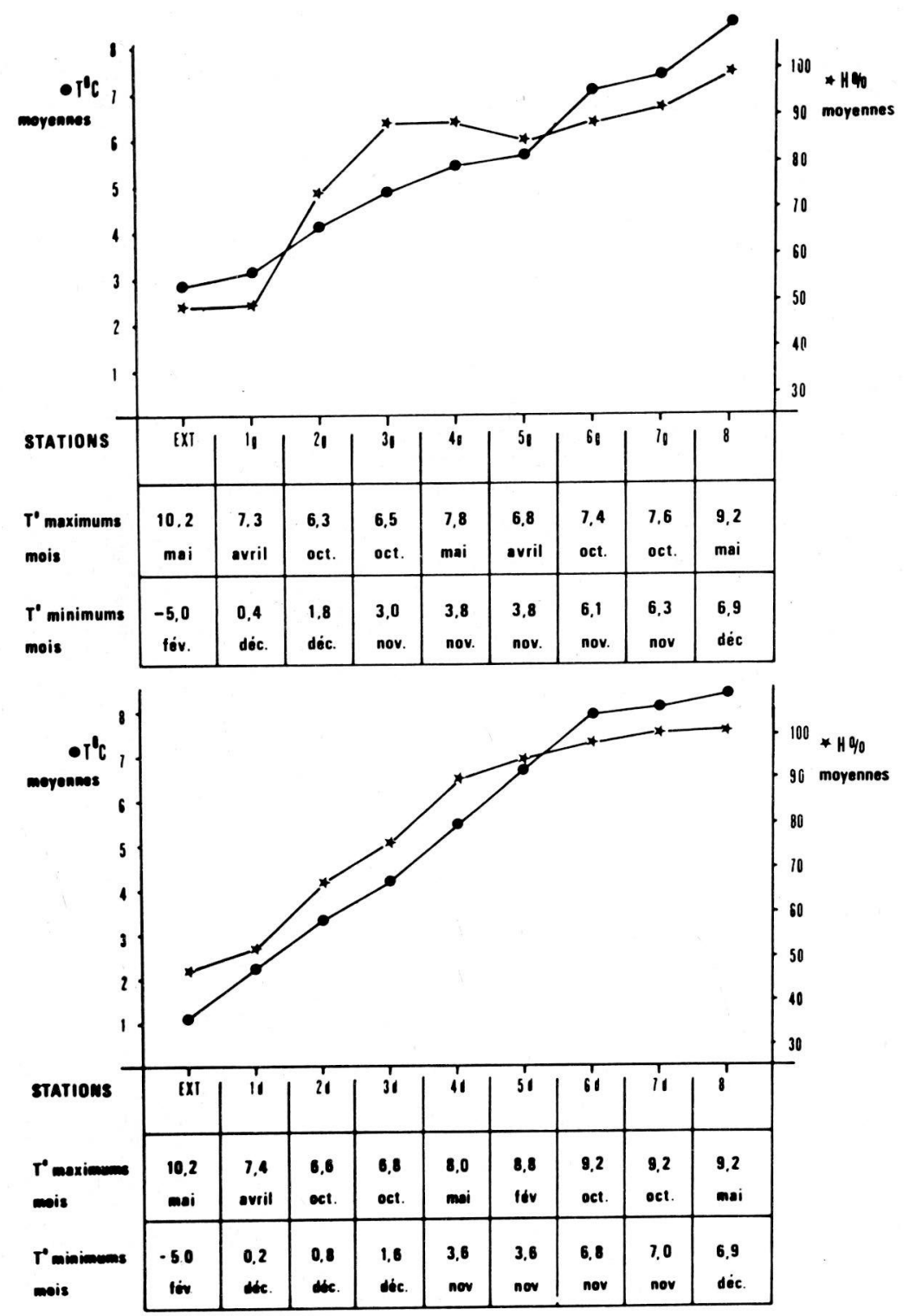

Fig. 3. Résumé des conditions climatologiques entre octobre 1974 et mai 1975. 


\section{RÉSULTATS}

\section{a. Distribution horizontale globale des 5 espèces}

Les figures $4 \mathrm{a}$ et $4 \mathrm{~b}$ nous montrent l'évolution de la faune pour chaque station selon les relevés; on remarque tout de suite une concentration de
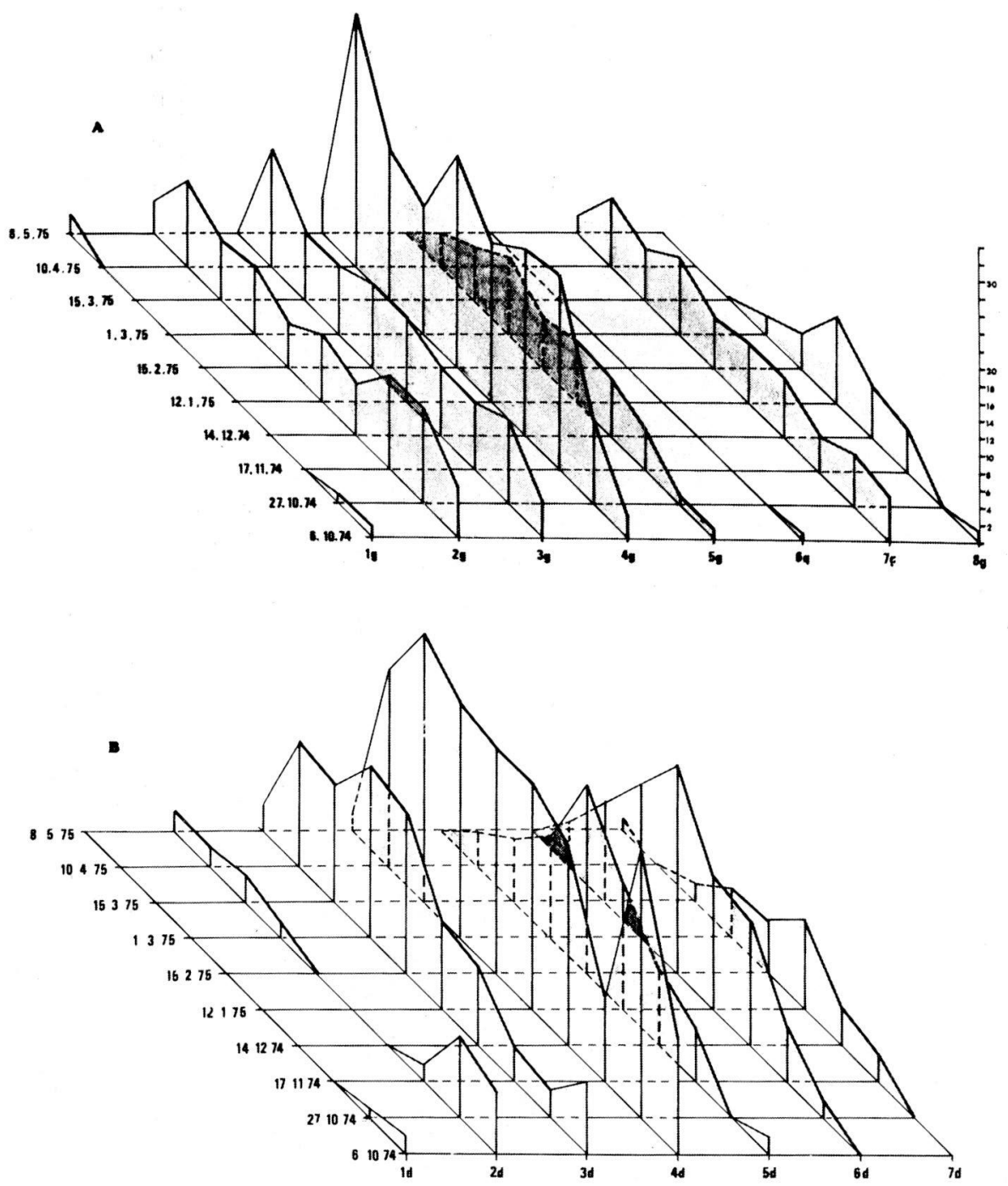

Fig. 4. a - Evolution mensuelle de la faune totale àl'intérieur des cadres, côte gauche, b - Idem pour le côté droit. 
la faune vers les stations $4 \mathrm{~g}$ et $4 \mathrm{~d}$. On voit également un certain mouvement vers le fond de la grotte pendant l'automne et l'hiver avec un mouvement contraire au printemps. A cause, peut-être, des mauvaises conditions météorologiques pendant les mois de mars et d'avril, un blocage du mouvement vers l'extérieur est à noter. La station $6 \mathrm{~g}$ est visiblement exceptionnelle et la non-habitation de cette parois se révèle intéressante.

\section{b. Distribution de chaque espèce pendant les 8 mois selon les stations ayant une densité maximum}

En prenant des stations de densité maximum (confirmé par les estimations hors des cadres) on peut voir plus clairement les mouvements saisonniers de chaque espèce dans l'écosystème. La figure 5 nous montre que les populations de chaque espèce se déplacent pendant leur séjour sous-terre mais de façon différente. Les moyennes pour les facteurs climatologiques montrent que les opiliones $N$. aurantiaca cherchent des régions plus chaudes et plus humides que les autres espèces. Les deux espèces de papillons restent toujours groupées vers les stations 4 , mais $T$. dubitata se déplace individuellement jusqu'à la station $8 \mathrm{~g}$ pendant l'hiver. Les araignées $M$. menardi ne pénètrent pas très loin dans la grotte, préférant des températures plus basses et évitant la condensation; elles ne dépassent jamais les stations 4 . La visite du 1.5.75 et le relevé du 8.5.75 nous montrent l'absence totale des papillons dans la grotte. Enfin, aucun opilione vivant n'a été trouvé le 31.5.75 par contre plusieurs cadavres moisis. Lors de cette visite on pouvait remarquer l'entrée en masse de plusieurs espèces de Diptères et des Trichoptères.

\section{c. Distribution verticale de chaque espèce}

Les cadres de $0,25 \mathrm{~m}^{2}$ nous permettent de préciser les microbiotopes préférés de chaque espèce. Un comptage de tous les relevés indépendemment des saisons et selon la morphologie des parois est résumé dans la figure 6 . Ce facteur n'a pas été le sujet d'étude précédemment et se révèle fort intéressant pour la Grotte de la Scierie.

\section{d. Les affinités entre les 5 espèces selon les cadres de $1 \mathrm{~m}^{2}$ (moyennes: 7 mois)}

Les calculs des affinités pour 174 relevés de $1 \mathrm{~m}^{2}$ (y compris les stations supplémentaires) entre oct. 1974 et avril 1975 se révèlent intéressants et confirment les résultats des préférences biotopiques verticales de chaque espèce (fig. 7). Mais, ils ne sont valables que pour l'écosystème car chaque cadre est composé de microbiotopes différents. Cependant la cohabitation de $M$. menardi, $N$. aurantiaca et $C$. pipiens semble concluante.

\section{e. Les affinités entre les 5 espèces selon les cadres de $0,25 \mathrm{~m}^{2}$ (moyennes: 7 mois)}

Comme pour les cadres de $1 \mathrm{~m}^{2}$ les relevés du mois de mai n'y sont pas inclus. En précisant les microbiotopes selon les critères de l'orientation spatiale 

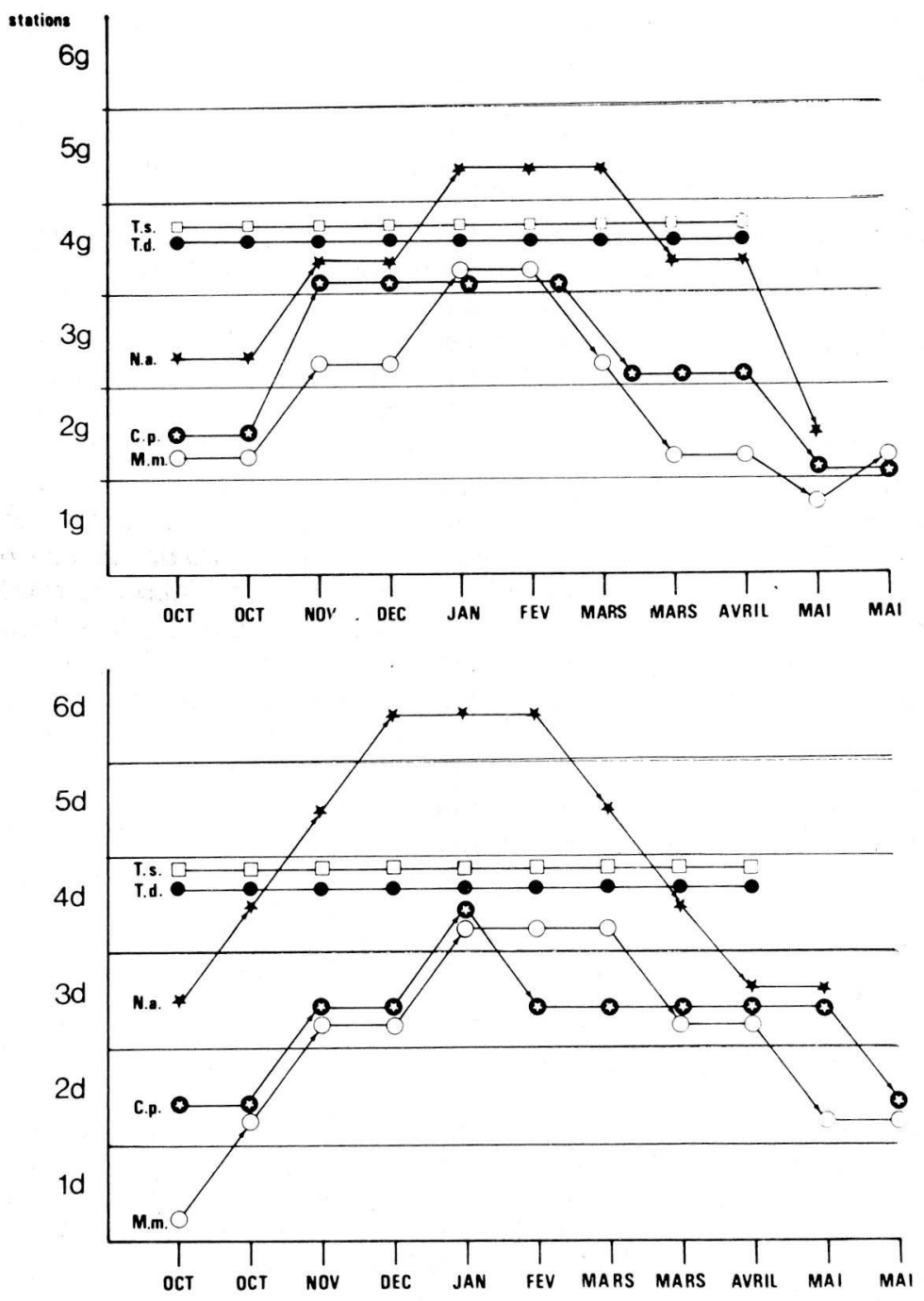

Fig. 5. Positions de la densité maximum pour chaque espèce selon les stations (oct. 1974 mai 1975). 


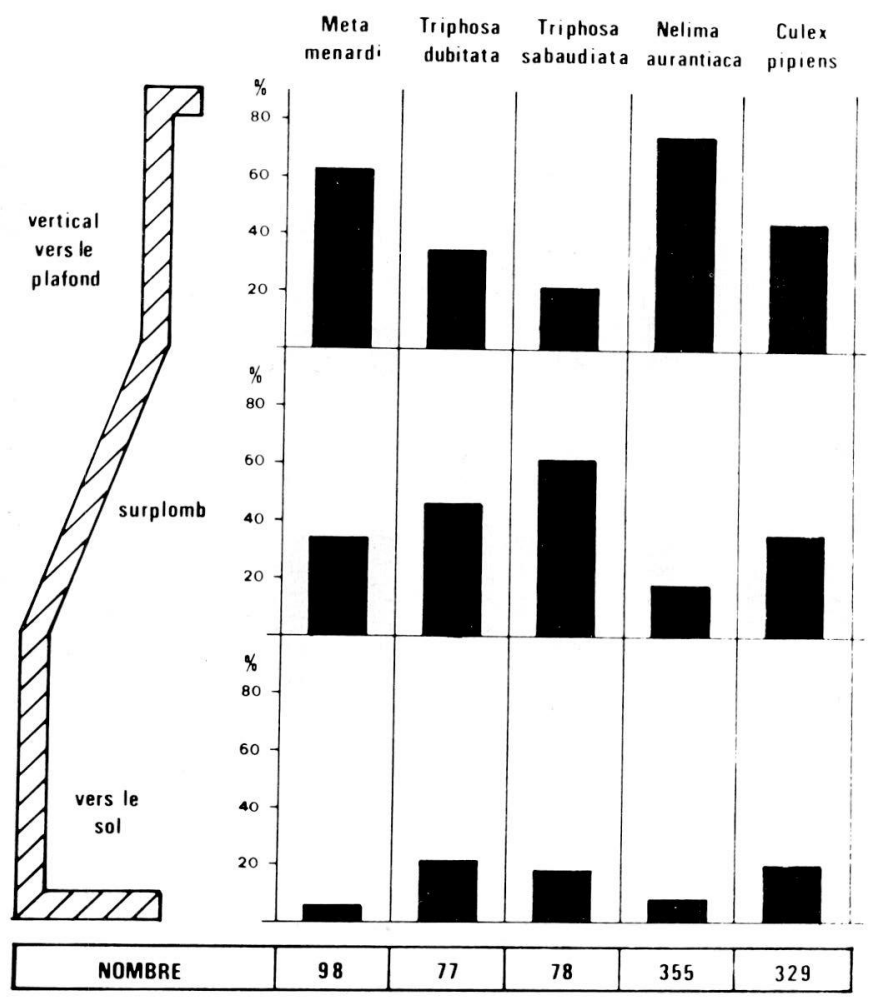

Fig. 6. Distribution verticale de chaque espèce en fonction de la morphologie des parois.

des parois, les affinités entre les paires d'espèces deviennent plus prononcées. La figure 8a nous montre les affinités (698 relevés) et, comparées aux résultats pour les cadres de $1 \mathrm{~m}^{2}$, certaines relations entre-specifiques sont, donc, accentuées. Par exemple le manque frappant d'affinités entre les papillons, $T$. dubitata et $T$. sabaudiata avec l'araignée $M$. menardi.

f. Evolution mensuelle des affinités selon les cadres de $0,25 \mathrm{~m}^{2}$

Les affinités entre chaque paire d'espèces selon les relevés mensuels confirment les mouvements saisonniers de chaque espèce au sein de l'écosystème. (fig. 8b). De même elles mettent en évidence le fait que ces espèces tendent à habiter toujours les mêmes types de microbiotopes. 


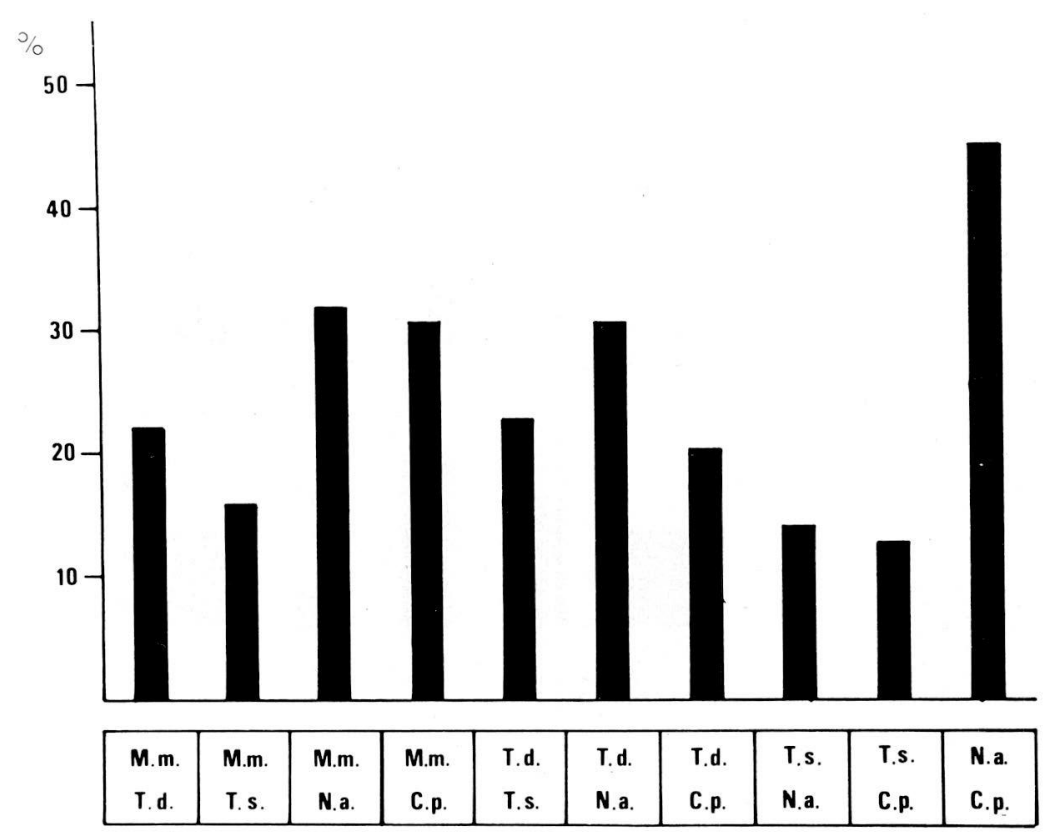

Fig. 7. Affinités entre les cinq espèces dans les cadres de $1 \mathrm{~m}^{2}$.

g. Recouvrement et orientation des papillons T. dubitata et T. sabaudiata (fig. 9)

Aucun cas de recouvrement de $T$. dubitata entre eux n'a été constaté dans cette grotte. Quelques cas de $T$. dubitata avec $T$. sabaudiata ont été observés entre décembre 1974 et février 1975 de même que de nombreux cas de $T$. sabaudiata entre eux. A partir de fin mars le nombre de cas isolés de $T$. sabaudiata a augmenté jusqu'à $100 \%$ : cependant ils se trouvaient groupés au sein des microbiotopes. Tous les papillons étaient très agités à partir de fin mars, courant sur les parois sous l'effet de ma lampe à carbure. La figure 9 nous montre également l'orientation de $T$. dubitata pendant les 8 mois d'observations. Il est à noter que je n'ai pas constaté de différences significatives de l'orientation causées par la lumière bien que la majorité des papillons étaient orientés vers le plafond. 


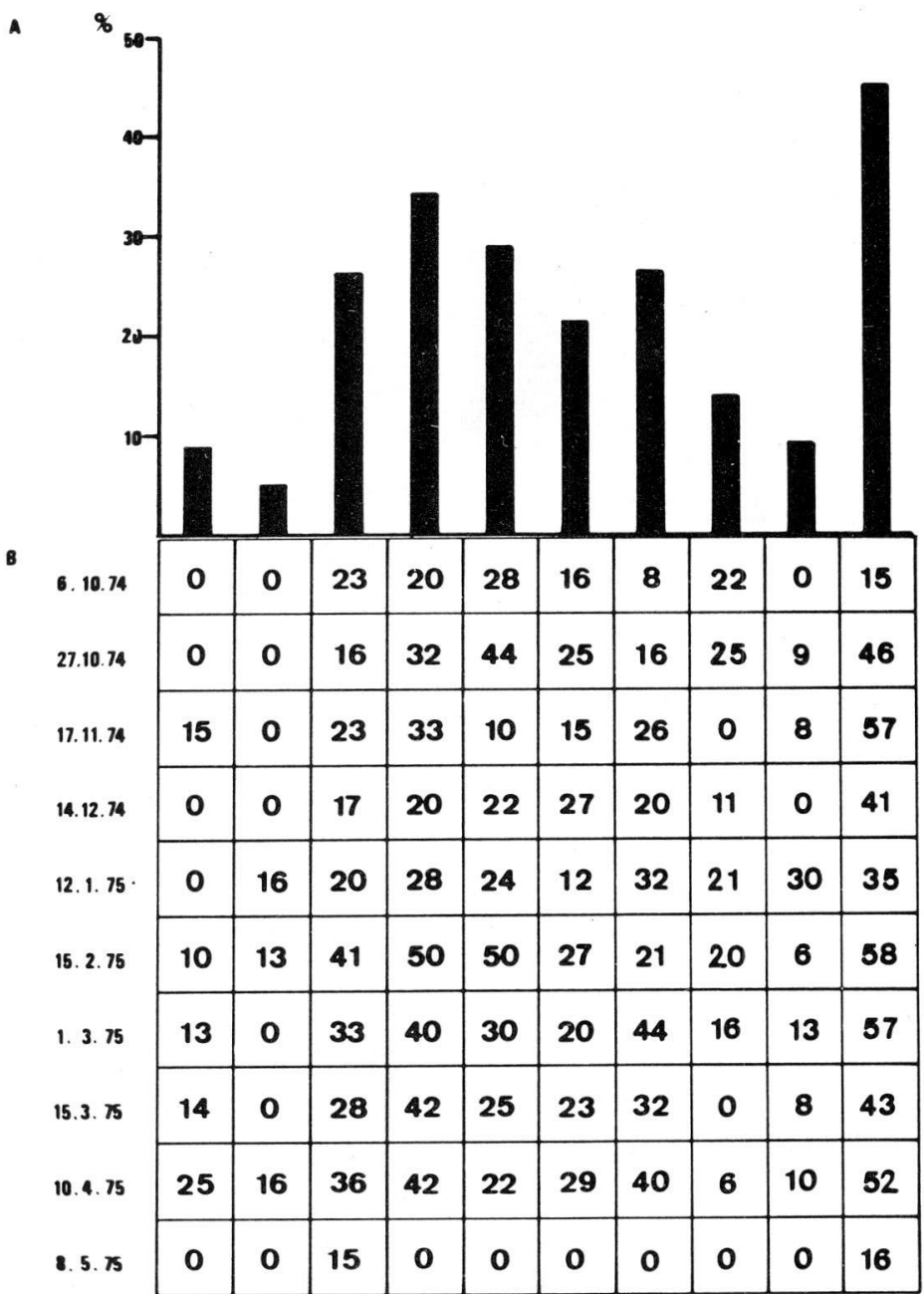

\begin{tabular}{|c|c|c|c|c|c|c|c|c|c|}
\hline T.d & I.s & แ. & C.p & $\begin{array}{l}\text { I.d } \\
\text { I.s }\end{array}$ & I. & $\begin{array}{l}\text { T.d } \\
c_{\text {. }}\end{array}$ & $\begin{array}{l}\text { T. } 8 \\
\text {. . }\end{array}$ & $\begin{array}{l}\text { T.s } \\
\text { C. }\end{array}$ & $\begin{array}{l}\text { m. } \\
\text { c.p }\end{array}$ \\
\hline
\end{tabular}

Fig. 8. a - Affinités entre les 5 espèces dans les cadres de $0,25 \mathrm{~m}^{2}$,

$\mathrm{b}$ - Evolution mensuelle des affinités entre les 5 espèces selon les cadres de $0,25 \mathrm{~m}^{2}$. 


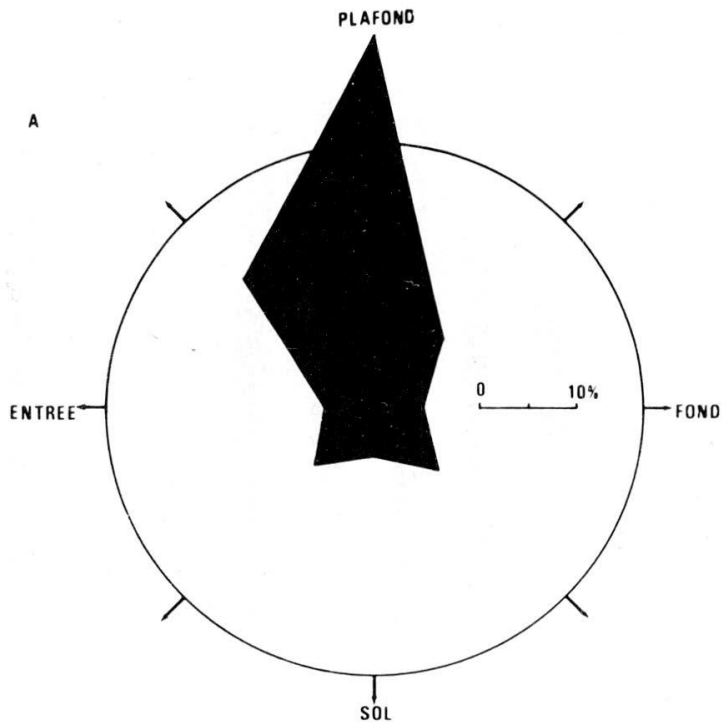

\begin{tabular}{|c|c|c|c|c|c|c|}
\hline \multirow{2}{*}{ MOIS } & \multicolumn{3}{|c|}{ Triphosa dubitata } & \multicolumn{3}{c|}{ Triphosa sabaud iata } \\
\cline { 2 - 7 } & $\begin{array}{c}\text { densite } / \mathrm{m}^{2} \\
\text { pour cadres } \\
\text { occupés }\end{array}$ & $\%$ isolés & $\begin{array}{c}\% \text { recouvre } \\
\text { ment avec } \\
\text { T. sabaudiat }\end{array}$ & $\begin{array}{c}\text { densite } / \mathrm{m}^{2} \\
\text { pour cadres } \\
\text { occupés }\end{array}$ & $\%$ isoles & $\begin{array}{c}\% \\
\text { recouvre } \\
\text { ment }\end{array}$ \\
\hline OCT & 6.0 & 100 & 0 & 7.0 & 80 & 20 \\
NOV & 6.8 & 100 & 0 & 8.0 & 100 & 0 \\
DEC & 7.2 & 80 & 20 & 9.0 & 0 & 100 \\
JAN & 6.0 & 90 & 10 & 5.5 & 10 & 90 \\
MEV & 104 & 75 & 25 & 7.0 & 5 & 95 \\
MARS & 52 & 100 & 0 & 90 & 10 & 90 \\
MARS & 50 & 100 & 0 & 40 & 100 & 0 \\
MVRIL & 4.4 & 100 & 0 & 25 & 100 & 0 \\
MAI & 0 & 0 & 0 & 0 & 0 & 0 \\
\hline
\end{tabular}

Fig. 9. a - Orientation de T. dubitata par rapport à l'entrée,

$\mathrm{b}-$ Recouvrements parmi les papillons entre octobre et avril. 


\section{DISCUSSION}

Pour une biocénose composée principalement d'espèces hivernantes on pourrait supposer que les liens trophiques ou d'autres serait moins nets que dans les biocénoses permanantes ou compétitives. Ainsi par définition, la faune pariétale (hiver) n'est en effet qu'une association plus ou moins définissable que par les besoins climatologiques communs aux espèce qui la composent.

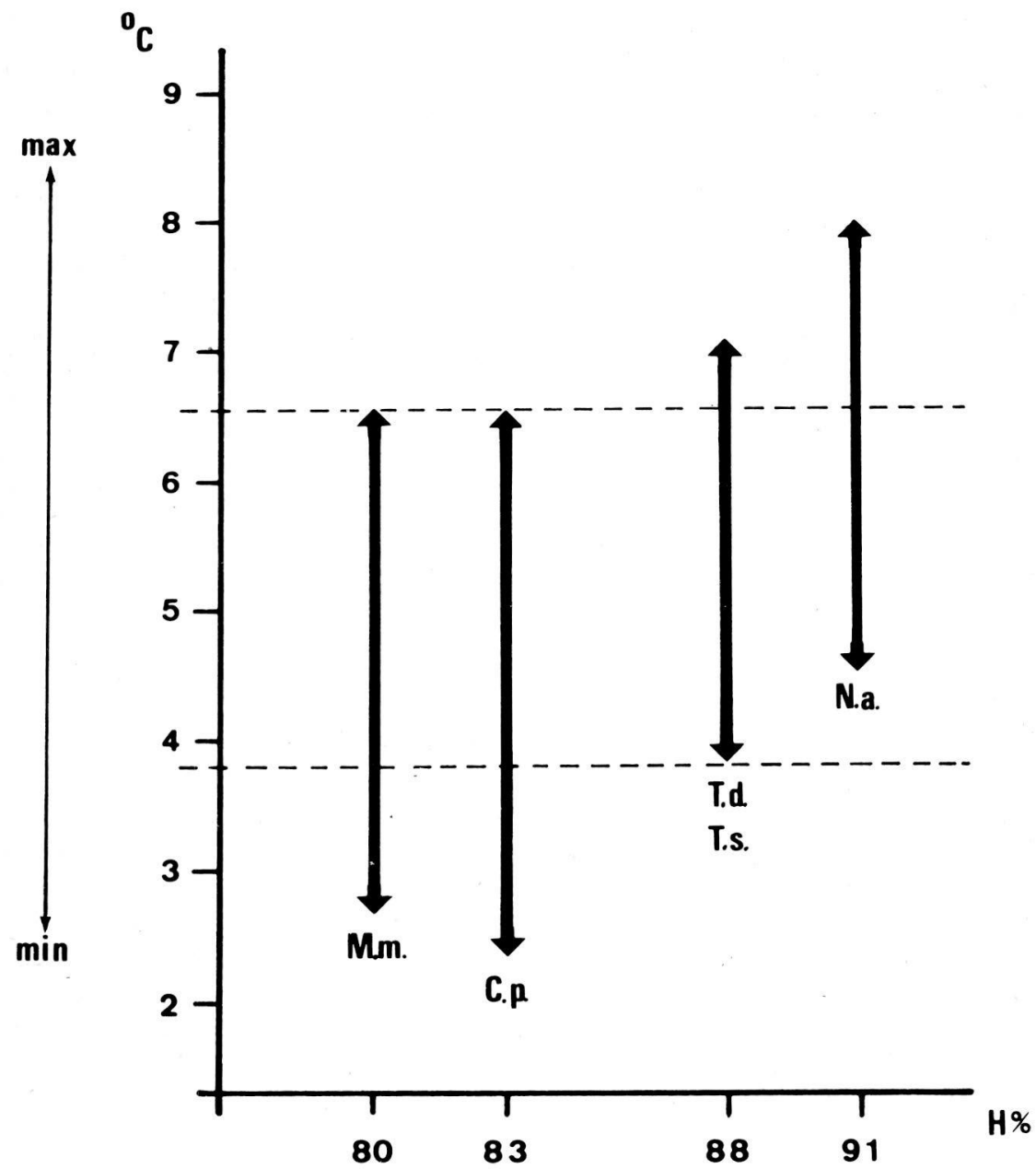

Fig. 10. Les constances maximums (flèches noires) pour chaque espèce mises en rapport avec les conditions climatologiques. (lignes pointillées $=$ variations de $\mathrm{T}^{\circ}$ pour les stations $4 \mathrm{~g}$ et $4 \mathrm{~d}$ entre oct. 74 et avril 75 ). 


\section{CONCLUSIONS}

Pendant huit mois d'observations il a été possible de démontrer le caractère actif des cinq espèces de la faune pariétale pendant leur séjour sous-terre. Leur distribution sur les parois de la Grotte de la Scierie est nettement influencés par les facteurs climatologiques et l'orientation des parois dans l'espace (verticales, surplombantes etc.). Les papillons $T$. sabaudiata sont distribués de façon contagieuses (agrégats") au sein des biotopes étudiés mais les autres espèces sont distribuées au hasard. Les araignées $M$. menardi et les deux espèces de papillons ont une distribution groupée dans l'écosystème. Par contre $C$. pipiens et $N$. aurantiaca semblent être distribués au hasard dans leurs biotopes et dans l'écosystème.

Cette première analyse nous montre que les araignées $M$. menardi (carnassières) et les deux espèces de papillons ont des affinités très peu prononcées, donc on serait tenté d'attribuer en partie une telle distribution à leur niveau trophique.

Les résultats permettent d'affirmer qu'une association entre ces cinq espèces dépend en majeure partie des facteurs climatologiques. Car, si l'on prend les constances maximum pour chaque espèce parmi les stations établies, et que l'on met en fonction les facteurs climatologiques des stations (fig. 10), on retrouve les stations $4 \mathrm{~g}$ et $4 \mathrm{~d}$ (stations les plus fortement peuplées).

Le caractère abiotique de la station $6 \mathrm{~g}$ illustre l'importance de l'orientation des parois.

Ces résultats seront peur-être modifiés par la suite car les relevés mensuels se poursuivent encore pendant une année. Enfin, aucune règle générale sur la distribution des cinq espèces étudiées ne peut être tirée car chaque grotte semble avoir ses particularités qui influencent la distribution de la faune pariétale.

\section{RÉSUMÉ}

Après 8 mois d'observations régulières dans la Grotte de la Scierie (HauteSavoie), il a été possible de décrire les mouvements et la distribution spatiale de Meta menardi (Araneae), Nelima aurantiaca (Opiliones), Triphosa dubitata et Triphosa sabaudiata (Lepidoptera), et Culex pipiens(Diptera) dans l'écosystème cavernicole. Bien qu'aucune règle générale ne puisse être établie il apparaît que la morphologie des parois et les conditions climatologiques influencent la distribution des 5 espèces au sein de l'écosystème cavernicole.

\section{BIBLIOGRAPHIE}

BOUVET, Y., M.-J. TURQUIN, C. BORNARD, S. DESVIGNES et P. NOTTEGHEM, 1974 - Quelques aspects de l'écologie et de la biologie de Triphosa et Scoliopteryx, Lépidoptères cavernicoles. Ann. Spéléol., 29, 2, 229-236. 
DAJOZ, R., 1971 - Précis d'Ecologie. Ed. DUNOD Paris, pp. 434.

GRAHAM, R. E., 1968 - Spatial biometrica of subterranean Triphosa haesitata (Lepidoptera: Geometridae). Caves and Karst, 10, 3, 21-29.

MOTAS, C., V. DECOU et A. BURGHELE, 1967 - Sur l'association pariétale des grottes d'Olténie (Roumanie). Ann. Spéléol., 22, 3, 475-522.

ROUBAUD, E., 1933 - Essai synthétique sur la vie du Moustique (Culex pipiens). L'évolution humaine et les adaptations biologiques du Moustique. Annal. Sci. Nat. Zool. (10) XVI.

TERCAFS, R., 1972 - Biomètrie spatiale dans l'écosystème souterrain: répartition de Meta menardi Latr. Int. J. Speleol. 4, 351-355.

VANDEL, A., 1964 - Biospéologie. La Biologie des Animaux Cavernicoles. Gauthier-Villars, Paris, pp. 619. 